\title{
Theory of hot matter and relativistic heavy-ion collisions (THOR)
}

\author{
Gert Aarts $^{1,2}$, Jörg Aichelin ${ }^{3}$, Marcus Bleicher ${ }^{4,5,6}$, Elena G. Ferreiro ${ }^{7}$, Laura Tolos ${ }^{8,9,10,11}$, Boris Tomášik ${ }^{12,13, a}$ (1) \\ ${ }^{1}$ Department of Physics, Swansea University,Singleton Campus, SA2 8PP Swansea, UK \\ 2 European Centre for Theoretical Studies in Nuclear Physics and Related Areas (ECT*) and Fondazione Bruno Kessler Strada delle Tabarelle \\ 286, 38123 Villazzano (TN), Italy \\ ${ }^{3}$ SUBATECH UMR 6457 (IMT Atlantique, Université de Nantes, IN2P3/CNRS), 4 Rue Alfred Kastler, F-44307 Nantes, France \\ ${ }^{4}$ Institut für Theoretische Physik, Goethe-University Frankfurt, Max-von-Laue-Str. 1, 60438 Frankfurt am Main, Germany \\ ${ }^{5}$ Helmholtz Forschungsakademie Hessen für FAIR (HFHF), Campus Frankfurt, Max-von-Laue-Str. 12, 60438 Frankfurt am Main, Germany \\ ${ }^{6}$ GSI Helmholtzzentrum für Schwerionenforschung GmbH, Planckstr. 1, 64291 Darmstadt, Germany \\ ${ }^{7}$ Instituto Galego de Fisica de Altas Enerxias - IGFAE, Universidade de Santiago de Compostela, E-15782 Santiago de Compostela, Spain \\ ${ }^{8}$ Institute of Space Sciences (ICE, CSIC), Campus UAB, Carrer de Can Magrans, 08193 Barcelona, Spain \\ ${ }^{9}$ Institut d'Estudis Espacials de Catalunya (IEEC), 08034 Barcelona, Spain \\ ${ }^{10}$ Faculty of Science and Technology, University of Stavanger, 4036 Stavanger, Norway \\ ${ }^{11}$ Frankfurt Institute for Advanced Studies, Ruth-Moufang-Str. 1, 60438 Frankfurt am Main, Germany \\ 12 Univerzita Mateja Bela, Tajovského 40, 97401 Banská Bystrica, Slovakia \\ ${ }^{13}$ Fakulta jaderná a fyzikálně inženýrská, České vysoké učení technické v Praze, Břehová 7, 11519 Praha 1, Czech Republic
}

Received: 12 April 2021 / Accepted: 20 April 2021 / Published online: 1 June 2021

(C) The Author(s), under exclusive licence to Società Italiana di Fisica and Springer-Verlag GmbH Germany, part of Springer Nature 2021

Communicated by Laura Tolos

This topical issue summarises some of the results realised thanks to the COST Action CA15213, which was running under the acronym THOR-short for Theory of hot matter and relativistic heavy-ion collisions-from November 2016 until April 2021.

Interesting progress has been made in explorations of hot, strongly-interacting matter with the help of heavy-ion collisions. Very precise high-statistics data furnished by experiments at the Large Hadron Collider at CERN and the Relativistic Heavy Ion Collider at Brookhaven National Laboratory, as well as a few smaller experiments, have definitely brought the field into the precision era. As a result, modern ideas need to be practically implemented in computable models and ultimately compared to data, with theoretical projects and results at all levels being vitally interconnected. From formal concepts based on underlying microscopic theories up to models aimed at simulations of real collision events and their outputs, outcomes at one level are applied at another and stimulate improvements there. For this reason, THOR worked very inclusively and supported a very broad range of theoretical projects that were connected with the exploration of hot and dense strongly-interacting matter.

The activities were organised within three Working Groups:

- WG1: Phases of strongly interacting matter;

\footnotetext{
a e-mail: boris.tomasik@ cern.ch (corresponding author)
}

- WG2: Dynamics of strongly interacting matter;

- WG3: Initial state and hard probes.

Even these Working Groups were deliberately formed in a rather broad manner in order to facilitate an exchange of ideas which would result from technically different approaches to solve the same problem.

After more than four years of running, 301 people participated in some way in THOR activities. Most of them were students at the Training Schools. THOR put a strong emphasis on the training of the future generation of experts and organised five schools in total. In addition, seven Scientific Meetings brought together scientists from one or more Working Groups and initiated new developments.

The most important activities, however, were the numerous Short Term Scientific Missions. THOR supported 73 such research visits. The papers collected in this issue of the European Physical Journal A are results that either came directly out of such collaboration or were crucially initiated by a THOR activity.

We would like to use this opportunity to express our gratitude to the COST Association-European Collaboration in Science and Technology - for the important support that helped to achieve a better understanding of hot and dense QCD matter. We are grateful for the welcoming and friendly support from our Science Officer, Dr. Fatima Bouchama, and from the Administrative Officers Cassia Azevedo and Milena 
Stojanova. We also thank our Grant Manager Jana Kunická for the smooth and prompt administration of this Action.

Finally, we thank to all colleagues who contributed to the Action and made its success possible.

Gert Aarts, Jörg Aichelin, Marcus Bleicher, Elena Ferreiro, Laura Tolos, and Boris Tomášik Guest editors

\section{Table of contents}

\section{Working group 1-Phases of strongly interacting} matter

(a) Electrical conductivity of the quark-gluon plasma: perspective from lattice $Q C D$ (G. Aarts and A. Nikolaev) [1]

(b) Thermal study of a coupled-channel system: a brief review (P. M. Lo) [2]

(c) Open-charm Euclidean correlators within heavymeson EFT interactions (G. Montaña, O. Kaczmarek, L. Tolos and A. Ramos) [3]

(d) Aspects of model dependence of $\eta^{\prime}-\eta$ complex treated by going beyond the isospin limit (D. Horvatić, D. Kekez and D. Klabučar) [4]

(e) Complex Langevin simulations and the QCD phase diagram: recent developments (F. Attanasio, B. Jäger and F. P. G. Ziegler) [5]

(f) Finite temperature $Q C D$ with $N_{f}=2+1+1$ Wilson twisted mass fermions at physical pion, strange and charm masses (A. Y. Kotov, M. P. Lombardo and A. M. Trunin) [6]

\section{Working Group 2-Dynamics of strongly interacting} matter

(a) Understanding the energy dependence of $B_{2}$ in heavy ion collisions: interplay of volume and spacemomentum correlations (V. Gaebel, M. Bonne, T. Reichert, A. Burnic, P. Hillmann and M. Bleicher) [7]

(b) Second virial coefficients of light nuclear clusters and their chemical freeze-out in nuclear collisions $(\mathrm{K}$. A. Bugaev, O. V. Vitiuk, B. E. Grinyuk, V. V. Sagun, N. S. Yakovenko, O. I. Ivanytskyi, G. M. Zinovjev, D. B. Blaschke, E. G. Nikonov, L. V. Bravina, E. E. Zabrodin, S. Kabana, S. V. Kuleshov, G. R. Farrar, E. S. Zherebtsova and A. V. Taranenko) [8]

(c) Deuteron and antideuteron coalescence in heavy-ion collisions: energy dependence of the formation geometry (A. Kittiratpattana, M. F. Wondrak, M. Hamzic, M. Bleicher, C. Herold and A. Limphirat) [9]

(d) Probing chemical freeze-out criteria in relativistic nuclear collisions with coarse grained transport simulations (T. Reichert, G. Inghirami and M. Bleicher) [10]

(e) Femtoscopy scales and particle production in the relativistic heavy ion collisions from $\mathrm{Au}+\mathrm{Au}$ at 200
$\mathrm{AGeV}$ to $\mathrm{Xe}+\mathrm{Xe}$ at $5.44 \mathrm{ATeV}$ within the integrated hydrokinetic model (V. M. Shapoval, M. D. Adzhymambetov and Y. M. Sinyukov) [11]

(f) Electromagnetic fields and directed flow in large and small colliding systems at ultrarelativistic energies (L. Oliva) [12]

(g) Emergent hydrodynamics in microscopic modeling of early stage of relativistic heavy-ion collisions (L. V. Bravina and E. E. Zabrodin) [13]

(h) Hadron production in elementary nucleon-nucleon reactions from low to ultra-relativistic energies ( V. Kireyeu, I. Grishmanovskii, V. Kolesnikov, V. Voronyuk and E. Bratkovskaya ) [14]

(i) The strange critical endpoint and isentropic trajectories in an extended PNJL model with eight Quark interactions (R. Câmara Pereira, J. Moreira and P. Costa ) [15]

(j) Evaluation of hypernuclei in relativistic ion collisions (N. Buyukcizmeci, A. S. Botvina, R. Ogul and M. Bleicher ) [16]

(k) Spin tensor and pseudo-gauges: from nuclear collisions to gravitational physics (E. Speranza and N. Weickgenannt) [17]

\section{Working Group 3-Initial state and hard probes}

(a) Thermal Field Theory in real-time formalism: concepts and applications for particle decays (T. Lundberg and R. Pasechnik) [18]

(b) Progress on 3+1D Glasma simulations (A. Ipp and D. I. Müller) [19]

(c) Contribution of the non-linear term in the BalitskyKovchegov equation to the nuclear structure functions (J. Cepila and M. Matas) [20]

(d) Photon induced processes: from ultraperipheral to semicentral heavy ion collisions (W. Schäfer) [21]

(e) Particle correlations from the initial state (T. Altinoluk and N. Armesto) [22]

\section{References}

1. G. Aarts, A. Nikolaev, Eur. Phys. J. A 57, 118 (2021). https://doi. org/10.1140/epja/s10050-021-00436-5

2. P.M. Lo, Eur. Phys. J. A 57, 60 (2021). https://doi.org/10.1140/ epja/s10050-021-00378-y

3. G. Montaña, O. Kaczmarek, L. Tolos, A. Ramos, Eur. Phys. J. A 56, 294 (2020). https://doi.org/10.1140/epja/s10050-020-00300-y

4. D. Horvatić, D. Kekez, D. Klabučar, Eur. Phys. J. A 56, 257 (2020). https://doi.org/10.1140/epja/s10050-020-00254-1

5. F. Attanasio, B. Jäger, F.P.G. Ziegler, Eur. Phys. J. A 56, 251 (2020). https://doi.org/10.1140/epja/s10050-020-00256-Z

6. A.Y. Kotov, M.P. Lombardo, A.M. Trunin, Eur. Phys. J. A 56, 203 (2020). https://doi.org/10.1140/epja/s10050-020-00216-7

7. V. Gaebel, M. Bonne, T. Reichert, A. Burnic, P. Hillmann, M. Bleicher, Eur. Phys. J. A 57, 55 (2021). https://doi.org/10.1140/epja/ s10050-020-00307-5 
8. K.A. Bugaev, O.V. Vitiuk, B.E. Grinyuk, V.V. Sagun, N.S. Yakovenko, O.I. Ivanytskyi, G.M. Zinovjev, D.B. Blaschke, E.G. Nikonov, L.V. Bravina, E.E. Zabrodin, S. Kabana, S.V. Kuleshov, G.R. Farrar, E.S. Zherebtsova, A.V. Taranenko, Eur. Phys. J. A 56, 293 (2020). https://doi.org/10.1140/epja/s10050-020-00296-5

9. A. Kittiratpattana, M.F. Wondrak, M. Hamzic, M. Bleicher, C. Herold, A. Limphirat, Eur. Phys. J. A 56, 274 (2020). https://doi. org/10.1140/epja/s10050-020-00269-8

10. T. Reichert, G. Inghirami, M. Bleicher, Eur. Phys. J. A 56, 267 (2020). https://doi.org/10.1140/epja/s10050-020-00273-y

11. V.M. Shapoval, M.D. Adzhymambetov, Y.M. Sinyukov, Eur. Phys. J. A 56, 260 (2020). https://doi.org/10.1140/epja/ s10050-020-00266-x

12. L. Oliva, Eur. Phys. J. A 56, 255 (2020). https://doi.org/10.1140/ epja/s10050-020-00260-3

13. L.V. Bravina, E.E. Zabrodin, Eur. Phys. J. A 56, 253 (2020). https:// doi.org/10.1140/epja/s10050-020-00265-y

14. V. Kireyeu, I. Grishmanovskii, V. Kolesnikov, V. Voronyuk, E. Bratkovskaya, Eur. Phys. J. A 56, 223 (2020). https://doi.org/10. 1140/epja/s10050-020-00232-7
15. R. Câmara Pereira, J. Moreira, P. Costa, Eur. Phys. J. A 56, 214 (2020). https://doi.org/10.1140/epja/s10050-020-00223-8

16. N. Buyukcizmeci, A.S. Botvina, R. Ogul, M. Bleicher, Eur. Phys. J. A 56, 210 (2020). https://doi.org/10.1140/epja/ s10050-020-00217-6

17. E. Speranza, N. Weickgenannt, Eur. Phys. J. A 57, 155 (2021). https://doi.org/10.1140/epja/s10050-021-00455-2

18. T. Lundberg, R. Pasechnik, Eur. Phys. J. A 57, 71 (2021). https:// doi.org/10.1140/epja/s10050-020-00288-5

19. A. Ipp, D.I. Müller, Eur. Phys. J. A 56, 243 (2020). https://doi.org/ 10.1140/epja/s10050-020-00241-6

20. J. Cepila, M. Matas, Eur. Phys. J. A 56, 232 (2020). https://doi.org/ 10.1140/epja/s10050-020-00243-4

21. W. Schäfer, Eur. Phys. J. A 56, 231 (2020). https://doi.org/10.1140/ epja/s10050-020-00231-8

22. T. Altinoluk, N. Armesto, Eur. Phys. J. A 56, 215 (2020). https:// doi.org/10.1140/epja/s10050-020-00225-6 